\title{
Elucidation of clinicopathological factors associated with improved clinical outcomes for carinal pneumonectomy in T4 non-small cell lung cancer
}

\author{
Ryuichi Waseda, So Miyahara, Toshihiko Moroga, Toshihiko Sato, Takeshi Shiraishi, Akinori Iwasaki \\ Department of General Thoracic, Breast, and Pediatric Surgery, Fukuoka University, Fukuoka, Japan \\ Contributions: (I) Conception and design: R Waseda; (II) Administrative support: R Waseda; (III) Provision of study materials or patients: R \\ Waseda, T Moroga; (IV) Collection and assembly of data: R Waseda, S Miyahara; (V) Data analysis and interpretation: R Waseda, S Miyahara; (VI) \\ Manuscript writing: All authors; (VII) Final approval of manuscript: All authors. \\ Correspondence to: Ryuichi Waseda, MD, PhD. 7-45-1 Nanakuma, Jonan-ku, Fukuoka, Japan. Email: wryuichi0119@fukuoka-u.ac.jp.
}

\begin{abstract}
Background: Surgical resection remains the gold standard of care for localized cancers. We retrospectively evaluated the efficacy and safety of carinal pneumonectomy for T4 non-small cell lung cancer (NSCLC), with a focus on the effect of changes in our treatment strategy on the outcome of the procedure over time at a single institution.
\end{abstract}

Methods: The study population consisted of consecutive patients with T4 NSCLC who underwent carinal pneumonectomy at our facility between the period of January 1993 and December 2016.

Results: Sixteen patients who underwent carinal pneumonectomy for T4 NSCLC were enrolled. Seven patients were operated before 2000 (the pre-2000 group) and nine were after 2000 (the post-2000 group). The multimodal oncological approach was more frequently used in the post-2000 group (6 patients, $66.6 \%$ in the post-2000 group $v s$. one patient, $14.3 \%$ in the pre-2000 group, $\mathrm{P}=0.04)$. Two patients $(12.5 \%)$, both in the pre-2000 group, showed 30-day mortality. In-hospital mortality was found in four patients (25\%), three in the pre-2000 group and one in the post-2000. Nine patients (56.4\%) developed major postoperative complications. Overall survival for the entire cohort was $61.4 \%$ at 1 -year, $54.5 \%$ at 3 -year, and $39.0 \%$ at 5 -year. Overall survival for the post-2000 group was $88.9 \%$ at 1 -year, $77.8 \%$ at 3 -year, and $51.9 \%$ at 5 -year. Overall survival for the post-2000 group was significantly superior to that of the pre-2000 group ( $\mathrm{P}=0.04)$. Univariate and multivariate analysis for clinicopathological factors influencing overall survival demonstrated that the multimodal approach had a significant positive impact $(\mathrm{P}=0.02)$.

Conclusions: In highly selected patients, carinal pneumonectomy for T4 NSCLC could be performed with acceptable postoperative outcomes and favorable survival outcomes. Multimodal approach including salvage surgery after definitive chemoradiation is likely to be associated with good long-term survival.

Keywords: Carinal pneumonectomy; non-small cell lung cancer (NSCLC); T4; multimodal therapy

Received: 15 December 2019. Accepted: 02 March 2020; Published: 10 October 2020.

doi: $10.21037 /$ shc.2020.03.06

View this article at: http://dx.doi.org/10.21037/shc.2020.03.06

\section{Introduction}

Surgical resection remains the gold standard of care for localized disease in the therapy for non-small cell lung cancer (NSCLC). Over the past half-century, various advances have been made in the field of surgical therapy including patient selection and perioperative management. Within this context, pneumonectomy is still considered to involve several challenges associated with high postoperative morbidity and mortality (1). On the other hand, pneumonectomy is not always sufficient for the radical resection of $\mathrm{T} 4$ diseases extending to the mediastinal structures, and extended pneumonectomy (EP) 
is required for particular T4 diseases. Borri et al. defined EP as pneumonectomy associated with one or more of the following structures: superior vena cava, tracheal carina, left atrium, aorta, chest wall, or diaphragm (2). Although all EP procedures are challenging and have higher postoperative risks than conventional pneumonectomy, the most technically demanding EP procedure is carinal pneumonectomy $(\mathrm{CP})$ which requires significant experience in treatment strategies and advanced skills to achieve desirable outcomes $(3,4)$.

In the present study, we retrospectively evaluated the efficacy and safety of CP for T4 NSCLC, with a focus on changes in our strategy and outcome of the procedure over time in a single institution.

\section{Methods}

The present study was approved by the Institutional Review Board of Fukuoka University Hospital (approval number: H19-12-003). This article was written according to the STROBE (Strengthening the reporting of observational studies in epidemiology).

\section{Patients}

The study population consisted of consecutive patients with T4 NSCLC who underwent CP at the department of General Thoracic, Breast and Pediatric Surgery, Fukuoka University Hospital between January 1993 and December 2016. Patients with adenoid cystic carcinoma, carcinoid, or other malignancies were excluded from this study.

\section{Preoperative evaluation}

All patients were uniformly staged with TNM version 8 (any previous TNM classifications were retrospectively converted to version 8) on the basis of enhanced computed tomography (CT) scan of the chest and the upper abdomen, brain magnetic resonance imaging (MRI), and bronchoscopy. Positron emission tomography using ${ }^{18} \mathrm{~F}$-fluorodeoxyglucose combined with CT (PET-CT) was regularly used after 2003. Invasive mediastinal lymph node (LN) investigation was performed if mediastinal $\mathrm{LN}$ metastases were suspected on CT or PET-CT. Patients with confirmed metastasis only to single-level N2 LNs (ipsilateral mediastinal and/or subcarinal) were not considered to be a contraindication for radical surgical therapy.

Functional tolerance was evaluated using spirometry, blood gas analysis, echocardiography, and lung ventilationperfusion scan. A predicted forced expiratory volume less than $800 \mathrm{~mL}$ was considered an absolute contraindication to surgery.

An oncological treatment strategy for each patient was discussed thoroughly and was individualized by a multidisciplinary lung cancer team, and the indication for carinal pneumonectomy was decided carefully.

\section{Intraoperative management and surgical technique}

All included patients underwent curative-intent surgery. As anesthetic techniques, double-lumen endotracheal tubes were used, and cross-field ventilation with a single lumen endotracheal tube was started just after transection of the main bronchus of the preserved lung. In several cases, highfrequency jet ventilation was used during anastomosis. The optimal surgical approach was selected by the surgical team in each case. Our suturing technique involved hybrid anastomosis of continuous running and interrupted fashion. The deep half of anastomosis including the membranous part of the airway was performed in a continuous fashion using a 4-0 non-absorbable monofilament suture; the remaining half was completed in an interrupted fashion using a 4-0 absorbable monofilament suture. To achieve tension-free anastomosis, various release maneuvers were performed. Anastomoses were usually covered with one or more vascularized tissue flaps. Additional procedures associated with CP such as resection of mediastinal structures were performed as required.

\section{Postoperative outcome}

Postoperative mortality and morbidity were recorded. Perioperative morbidity was evaluated according to the Clavien-Dindo Classification (5).

\section{Follow-up}

All patients were monitored every three months for the first year from the end of the treatment and then every 6 months. Follow-up data were retrieved from medical records, clinical charts, and imaging reports (CT, MRI or PET/CT). Survival data up until October 2019 was used in the analysis. 
Table 1 Patient characteristics

\begin{tabular}{|c|c|c|c|c|}
\hline Characteristics & Overall $(n=16)$ & Pre-2000 $(n=7)$ & Post-2000 (n=9) & $P$ \\
\hline Sex, n (\%) & & & & 1 \\
\hline Male & $16(100.0)$ & $7(100.0)$ & $9(100.0)$ & \\
\hline Female & $0(0.0)$ & $0(0.0)$ & $0(0.0)$ & \\
\hline Squamous cell carcinoma & $7(43.8)$ & $3(42.9)$ & $4(44.4)$ & \\
\hline Adenocarcinoma & $7(43.8)$ & $3(42.9)$ & $4(44.4)$ & \\
\hline Large-cell carcinoma & $2(12.4)$ & $1(14.2)$ & $1(11.2)$ & \\
\hline Pathologic nodal status, n (\%) & & & & 0.46 \\
\hline 2 & $9(56.3)$ & $5(71.4)$ & $4(44.4)$ & \\
\hline Oncologic treatment strategy, n (\%) & & & & 0.27 \\
\hline Surgery alone & $9(56.2)$ & $6(85.7)$ & $3(33.4)$ & \\
\hline Surgery + adjuvant CT & $3(18.7)$ & $1(14.3)$ & $2(22.2)$ & \\
\hline Induction CT + surgery + adjuvant CT & $1(6.3)$ & $0(0.0)$ & $1(11.1)$ & \\
\hline Induction CRT + surgery & $2(12.5)$ & $0(0.0)$ & $2(22.2)$ & \\
\hline Definitive CRT + surgery & $1(6.3)$ & $0(0.0)$ & $1(11.1)$ & \\
\hline
\end{tabular}

SD, standard deviation; CT, chemotherapy; CRT, chemoradiotherapy.

\section{Statistical analysis}

All statistical analyses were performed using the statistical software package SPSS, version 24.0 (IBM-SPSS Inc, Armonk, NY). Survival was calculated from the day of the surgery using the Kaplan-Meier method. Overall survival (OS) in this study was defined as the period from the date of the surgery to the date of patient death due to any cause or the last patient follow-up date. The univariate and multivariate Cox regression analysis was used to estimate hazard ratios (HRs) and $95 \%$ confidence interval (CI) for clinical factors influencing OS. Statistical significance was defined as $\mathrm{P}<0.05$ (two-sided).

To analyze the effect of increased clinical experience on treatment outcomes, the study was divided into two time periods: a pre-2000 group and a post-2000 group. The differences between the two time periods were calculated using either One-way analysis of variance (ANOVA) or Chisquare test. Survival differences between the groups were also determined using the log-rank test.

\section{Results}

Over the course of the study period, 2,585 surgical resections for lung cancer including 126 pneumonectomies were performed. Of those, 16 patients who underwent carinal pneumonectomy for T4 NSCLC were enrolled. Table 1 shows patient characteristics. All patients were male with a mean age of 62.4 years (range, $41-74$ years). The pre-2000 group included seven patients, and nine patients comprised the post-2000 group. Nine patients were diagnosed with the N2 disease, the majority of which were due to mediastinal nodes directly involved with the primary tumor.

\section{Oncological treatment strategy}

Twelve patients had upfront surgical therapy, two patients received induction chemoradiotherapy, and one patient received induction chemotherapy. One patient underwent definitive chemoradiation at a regional hospital. This 
Table 2 Summary regarding surgical procedure

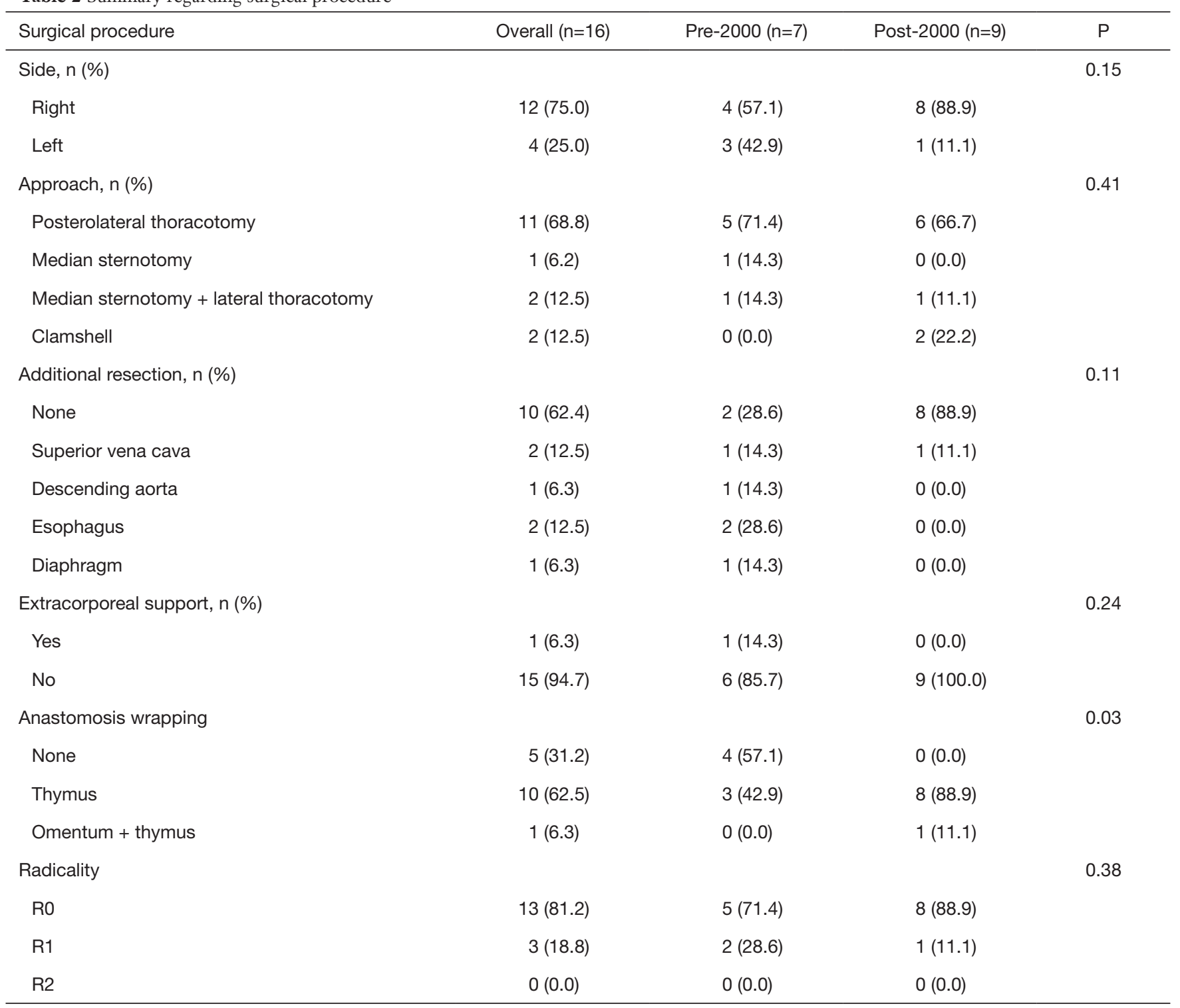

patient had progressive disease and was referred to us for palliative airway stenting after having undergone previous therapy. His general condition dramatically recovered, and our systemic evaluation revealed that his disease was still localized. Therefore, our multidisciplinary lung cancer team decided to perform salvage surgery in this patient. Adjuvant therapy was performed in four patients. A patient in the pre2000 group received adjuvant chemotherapy, and the other six in this group underwent surgery alone. Consequently, the multimodal treatment approach was more frequently used in the post- 2000 group (6/9 patients or $66.6 \%$ in the post-2000 group vs. $1 / 7$ patient or $14.3 \%$ in the pre-2000 group, $\mathrm{P}=0.04)$.

\section{Surgical procedure}

Data regarding surgical procedures are summarized in Table 2. Right CP was performed in 12 patients (75\%). The most frequently used surgical approach for the right CP was posterolateral thoracotomy (10 patients, $83 \%$ ). On the other hand, median sternotomy with or without lateral thoracotomy was frequently used for left CP (3 patients, $75 \%)$. The clamshell approach was adopted to two right $\mathrm{CP}$ procedures to perform left hilar release or to perform 
Table 3 Summary regarding postoperative outcomes

\begin{tabular}{|c|c|c|c|c|}
\hline Postoperative outcomes & Overall $(n=16)$ & Pre-2000 $(n=7)$ & Post-2000 (n=9) & $\mathrm{P}$ \\
\hline Yes & $2(12.5)$ & $2(28.6)$ & $0(0.0)$ & \\
\hline No & $14(87.5)$ & $5(71.4)$ & $9(100.0)$ & \\
\hline In-hospital mortality, n (\%) & & & & 0.15 \\
\hline No & $12(75.0)$ & $4(57.4)$ & $8(88.9)$ & \\
\hline Morbidity (more than grade III), n (\%) & & & & 0.37 \\
\hline None & $7(43.6)$ & $2(28.6)$ & $5(55.6)$ & \\
\hline Respiratory failure & $4(25.0)$ & $2(28.6)$ & $2(22.2)$ & \\
\hline Anastomotic dehiscence & $1(6.3)$ & $1(14.3)$ & $0(0.0)$ & \\
\hline Anastomotic stricture & $1(6.3)$ & $0(0.0)$ & $1(11.1)$ & \\
\hline
\end{tabular}

resection and reconstruction of superior vena cava (SVC). Additional resections were performed more frequently in the pre-2000 group than in the post-2000 group (4/7 patients or $71.4 \%$ in the pre-2000 vs. $1 / 9$ patients or $11.1 \%$ in the post-2000 group, $\mathrm{P}=0.01$ ). Extracorporeal membrane oxygenation (ECMO) was used only for the resection and reconstruction of the descending aorta. Anastomoses in the post-2000 group were wrapped with vascularized tissue flaps more frequently than in the pre-2000 group (9/9 patients or $100 \%$ in the post- 2000 group $v s .3 / 7$ patient, $42.9 \%$ in the pre-2000 group, $\mathrm{P}=0.01$ ). Thymus was normally used as the wrapping material, while a combination of omentum and thymus was used in a case of high-risk anastomosis that had received a full dose of radiotherapy. $\mathrm{R} 0$ resection was not achieved in three patients ( $3 / 16$ or $18.8 \%)$. Two patients had a positive microscopical margin of resected airways, and the resected muscle layer of the esophagus was microscopically positive in one patient.

\section{Postoperative outcomes}

Postoperative outcomes are summarized in Table 3. The 30day mortality was observed in two patients (2/16 or $12.5 \%)$. Both patients belonged to the pre-2000 group-one had a fatal ventricular arrhythmia just after the operation, and the other died on postoperative day 16 due to severe pneumonia. The post-2000 group did not show any 30day mortality. In-hospital mortality was observed in four patients $(4 / 16$ or $25 \%)$-three in the pre- 2000 group $(3 / 7$ or $42.6 \%)$ and one in the post- 2000 group (1/9 or $11.1 \%)$. Nine patients (9/16 or $56.4 \%)$ developed major postoperative complications. Four patients (4/16 or $25 \%)$ had severe respiratory failure, and three of them required tracheostomy. More seriously, two patients (2/16 or $12.5 \%)$ suffered acute respiratory distress syndrome (ARDS) which resulted in in-hospital mortality. Anastomosis related complications were observed in two patientsone with anastomotic dehiscence and another who developed anastomotic stricture. The dehiscence caused empyema; however, it was controlled by open window therapy. The stricture was observed in the patient who had undergone definitive chemoradiotherapy including $60 \mathrm{~Gy}$ of irradiation. The anastomosis was tense even after several release maneuvers due to long segmental resection. The anastomosis became necrotic two weeks after the operation; nevertheless, the combined flap comprised of omentum and thymus was evidently effective in avoiding any infectious complications. Eventually, the patient developed mild anastomotic stricture which required endoscopic balloon dilatation. While the overall postoperative mortality and morbidity were less in the post-2000 group than in the pre2000 group, the difference was not statistically significant. 


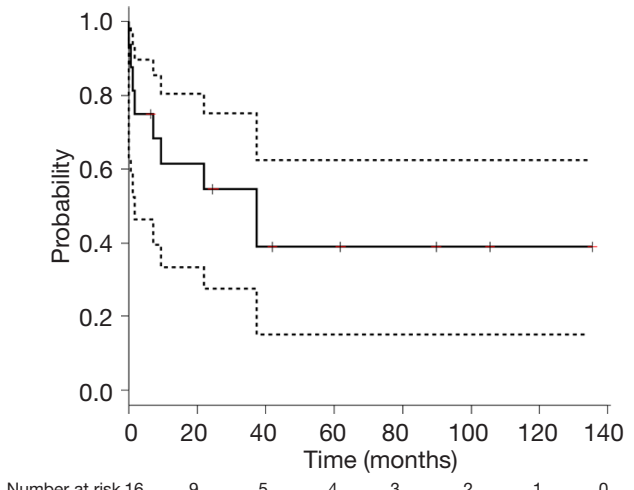

Figure 1 Kaplan-Meier curve (solid line) with 95\% CI (dotted line) for the overall survival of all 16 patients.

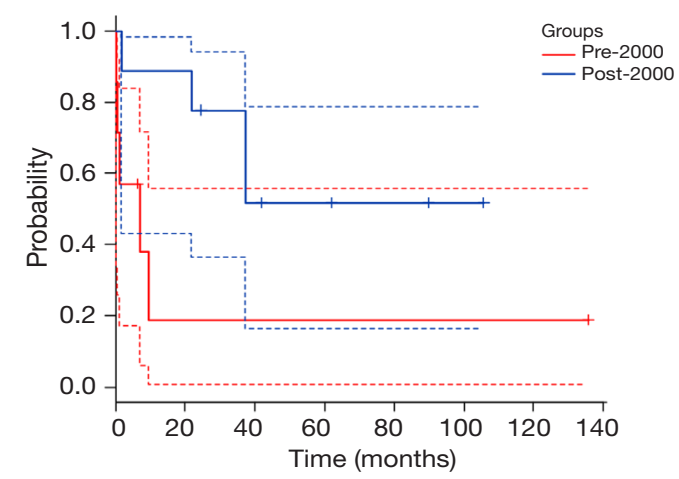

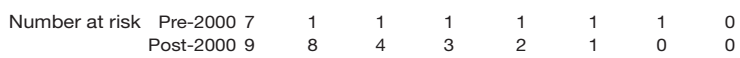

Figure 2 Kaplan-Meier curve (solid line) with 95\% CI (dotted line) for the overall survival according to treatment group.

\section{Survival outcomes}

The observed OS for the entire cohort was $61.4 \%$ at 1 -year (95\% CI: $33.3-80.5 \%$ ), $54.5 \%$ at 3-year (95\% CI: $27.4-$ $75.3 \%$ ), and $39 \%$ at 5 -year (95\%CI: 15.1-62.5\%) (Figure 1). The pre-2000 group showed OS of 19\% at 1-, 3- and 5-year (95\% CI: 9.2-55.6\%). The post-2000 group showed OS of $88.9 \%$ at 1 -year (95\% CI: $43.3-98.4 \%$ ), $77.8 \%$ at 3 -year (95\% CI: $36.5-93.9 \%$ ), and $51.9 \%$ at 5 -year (95\% CI: $16.4-$ $78.8 \%)$. The OS for the post-200 group was significantly superior to that of the pre-2000 group $(\mathrm{P}=0.04)$ (Figure 2).

Table 4 shows the Clinicopathological factors influencing OS. Univariate analysis identified the multimodal approach and the treatment in the post-2000 group as significantly associated with favorable prognosis $(\mathrm{P}=0.01$ and $\mathrm{P}=0.05$, respectively). Multivariate analysis on significant and marginally significant variables derived from the univariate analysis revealed that the multimodal approach was still a significant positive factor for $\mathrm{OS}(\mathrm{P}=0.02)$.

\section{Discussion}

In the present study, we reviewed a series of CPs performed for pathologically proven T4 NSCLC. Perioperatively, we report a $12.5 \% 30$-day mortality and a $25 \%$ in-hospital mortality. The long-term survival outcome was $54.5 \%$ at 3 -year and $39.0 \%$ at 5 -year. Furthermore, our institutional results for CPs performed after the year 2000 showed marked improvement in both the perioperative and the long-term outcomes, showing no 30-day mortality, $11.1 \%$ in-hospital mortality, $77.8 \% 3$-year OS, and 51.9\% 5-year OS.

Carinal pneumonectomy, as other EPs, continues to be a technically complex procedure with the potential for fatal surgical morbidity and mortality even in modern thoracic surgery. Since the early reports of experiences with carinal surgery including CP in the 1950s (6-8), many studies have been published originating mainly from the major thoracic centers worldwide (9-16). These studies have demonstrated clear improvements over the early results, with a $0-16 \%$ decrease in postoperative mortality and a $25-66.3 \%$ increase in 5-year OS. However, most studies were not limited to CP; other types of carinal surgery such as carinal lobectomy or carinal resection without lung resection were also included. Furthermore, a heterogeneous mix of diseases was examined including low-malignancy bronchogenic neoplasms and other benign diseases in addition to NSCLC. On the other hand, our study cohort exclusively consisted of T4 NSCLC patients who underwent CP solely for curative intent. These differences may have negatively impacted our operative and survival outcomes in comparison with other studies. A recent report by Costantino et al. described excellent operative mortality of $6.8 \%$; however, the mortality after CP was $12 \%$ in the same report (16). In comparison, Eichhorn et al. used a patient cohort very similar to our study cohort, and our post-2000 results are equivalent or better than their reported outcomes of 3\% 30-day mortality, $40 \% 3$-year OS, and $31 \%$ of 5 -year OS (12).

As numerous articles have described, patient selection, perioperative management including anesthetic technique, and surgical technique are key factors for achieving favorable short-term surgical and long-term oncologic outcomes after carinal surgery (3,4,9-16). Our outcomes showed clear improvements over the two decades of the study, and therefore analysis of these factors between the two study periods was performed. Preoperative functional 
Table 4 Results of univariate and multivariate analyses for clinicopathological factors influencing OS

\begin{tabular}{|c|c|c|c|c|c|c|}
\hline Factors & \multicolumn{3}{|c|}{ Univariate analysis } & \multicolumn{3}{|c|}{ Multivariate analysis } \\
\hline Age & 1.00 & $0.91-1.11$ & 0.98 & & & \\
\hline \multicolumn{7}{|l|}{ Side } \\
\hline Right & 1.00 & & & & & \\
\hline \multicolumn{7}{|l|}{$\mathrm{pN}$ status } \\
\hline $\mathrm{pNO} / 1$ & 1.00 & & & 1.00 & & \\
\hline pN2 & 2.75 & $0.67-11.31$ & 0.16 & 0.98 & $0.18-5.36$ & 0.98 \\
\hline \multicolumn{7}{|c|}{ Additional resection } \\
\hline \multicolumn{7}{|c|}{ Multimodal approach } \\
\hline No & 1.00 & & & 1.00 & & \\
\hline Yes & 0.07 & $0.01-0.58$ & 0.01 & 0.07 & $0.01-0.71$ & 0.02 \\
\hline \multicolumn{7}{|c|}{ Surgical radicality } \\
\hline Ro & 1.00 & & & 1.00 & & \\
\hline $\mathrm{R} 1$ & 4.51 & $0.89-22.8$ & 0.07 & 0.97 & $0.14-6.86$ & 0.97 \\
\hline \multicolumn{7}{|c|}{ Treatment group } \\
\hline Pre-2000 & 1.00 & & & 1.00 & & \\
\hline
\end{tabular}

OS, overall survival; $\mathrm{HR}$, hazard ratio; $\mathrm{Cl}$, confidence intervals.

assessment for CP has remained unchanged since 1993, and the same is also true for our preoperative oncologic evaluations. Therefore, there were no significant differences with regard to patient characteristics between the two study groups. Relatively few differences were noted in the actual treatments performed, for example, oncologic treatment strategy, additional extended resection other than carina, and anastomosis wrapping. In our opinion, the observed improvement in both perioperative and long-term outcomes cannot be sufficiently explained with just the differences in treatments.

The multimodal treatment approach was not indicated frequently despite locally advanced disease, especially in the pre-2000 study group. This is likely due to the preoperative determination of $\mathrm{N} 0$ disease in these patients. We strictly excluded pathologically proven multiple mediastinal LN positive patients as surgical candidates. Although more than
$90 \%$ of our patients had positive LNs in the final pathology, most cases were due to direct infiltration of centrally located primary tumors. The benefits of multimodal treatment for potentially resectable NSCLC have been a subject of great controversy as discussed by several studies, and significant survival benefits of neoadjuvant or adjuvant therapy for completely resected locally advanced NSCLC have not been observed $(17,18,19)$. Although a number of studies have reported pathologic LN status as a strong poor prognostic factor for CP, it did not influence OS significantly in the present study. With the identification of positive LNs likely due to the direct infiltration of the primary tumor, as mentioned above, the nodal status might represent just the size or location of primary tumors in this study rather than actual metastasis, thus explaining the lack of its effect on OS in this study.

Nevertheless, multimodal therapy has been used more 
frequently over the years at our institution. After 2000, half of our patients received some sort of therapy prior to surgery. Our analysis of clinicopathological factors influencing OS revealed that any type of multimodal approach had a strong positive impact on OS. However, since our multimodal approach has not been standardized, its advantages and disadvantages for CP should be carefully considered. Several reports have described that induction treatment prior to $\mathrm{CP}$ increases the risk of perioperative mortality and morbidity. Especially, a negative influence has been ascribed to preoperative radiotherapy for airway anastomoses. However, the negative influence of preoperative treatment was not reflected in our postoperative results. Even in a particular case of salvage surgery after a full dose of chemoradiotherapy, the fatal anastomotic complication was not observed. The advantages of preoperative therapy include not only systemic effects to control LNs or distant metastases but also local effects to reduce the volume of a target lesion. The local effects could diminish various operative risks, especially anastomotic complications in CP. Minimizing the extent of resection leads to a reduction in the tension of anastomosis, and also could preserve the blood supply to anastomosis from the surrounding tissues. The fact that additional extended resections other than carina were performed less frequently in the post-2000 group might be a result of preoperative treatment, though the changing bias in our patient selection over time should also be considered. Nevertheless, our postoperative mortality and morbidity have distinctly improved after 2000, although not significantly.

While clear evidence to support the efficacy of anastomosis wrapping has not been reported, several institutions including ours have routinely performed any anastomosis wrapping after 2000 (3,4,6-16). Our core objective with anastomotic wrapping is to isolate the anastomotic site from the chest cavity with an adequate quantity of vascularized tissue flaps. Although thymus is used routinely as a wrapping material at our institution, and the combination flap of thymus and omentum may be better suited in the cases of high-risk anastomosis. The combination wrapping was indeed effective in avoiding the fatal anastomotic problems especially in the case involving 60 Gy of chemoradiation. Constantino et al. have described in detail the concepts of anastomotic wrapping, the use of vascularized tissues in anastomoses, and the application of omentum in high-risk anastomoses with a history of radiotherapy (16).

In recent years, substantial progress has been made in the therapy of NSCLC. For example, target therapy and immunotherapy have emerged as new modes of systemic therapy, whereas particle beam therapy and stereotactic body radiotherapy are gaining attention in radiation therapy. The role of surgery in the therapy of NSCLC has undergone a change, although surgical resection remains the gold standard of care for localized disease. A multimodal approach is now commonly performed in locally advanced NSCLC. Furthermore, salvage surgery after definitive chemoradiotherapy or immunotherapy is a viable option $(20,21)$. Although highly established operative skills and managements should be required to achieve promising results in the particular salvage situations, favorable longterm results can be expected due to positive effects of patient selection instead of the operative difficulties and risks. Indeed, an excellent survival outcome after 2000 was demonstrated in the present study, with $77.8 \%$ of 3 -year OS and $51.9 \%$ of 5 -year OS.

The main limitation of this study is its single institutional and retrospective nature. Our treatment strategy including surgical techniques and perioperative management evolved over the study period. This change could have introduced positive bias and improved the outcome of patients operated in the later time period. Another limitation is the small sample size, which decreases the power of any statistical findings. However, given the technically demanding nature of $\mathrm{CP}$ and the limited worldwide experiences with $\mathrm{CP}$ in $\mathrm{T} 4$ NSCLC, we believe that the findings presented here are of great importance for a better understanding of the current status of CP for T4 NSCLC.

\section{Conclusions}

In conclusion, CP for T4 NSCLC could be performed for highly selected patients with acceptable postoperative outcomes and favorable survival outcomes. Multimodal approach including salvage surgery after definitive chemoradiation might be associated with good long-term survival. However, well-established operative skills and optimal surgical management is necessary to perform $\mathrm{CP}$ safely as a part of multimodal therapy. Therefore, CP for T4 NSCLC should be performed only in the thoracic centers with experience in this procedure.

\section{Acknowledgments}

Funding: The study was supported by a grant from SougouKagaku Kennkyu team of Fukuoka University (181043). 


\section{Footnote}

Provenance and Peer Review: This article was commissioned by the Guest Editors (Lorenzo Spaggiari and Luca Bertolaccini) for the series "The Role of Pneumonectomy in Thoracic Surgery in The Third Millennium" published in Shanghai Chest. The article has undergone external peer review.

Conflicts of Interest: All authors have completed the ICMJE uniform disclosure form (available at http://dx.doi. org/10.21037/shc.2020.03.06). The series “The Role of Pneumonectomy in Thoracic Surgery in The Third Millennium" was commissioned by the editorial office without any funding or sponsorship. The authors have no other conflicts of interest to declare.

Ethical Statement: The authors are accountable for all aspects of the work in ensuring that questions related to the accuracy or integrity of any part of the work are appropriately investigated and resolved. This study was conducted in accordance with the Declaration of Helsinki (as revised in 2013). The present study was approved by the Institutional Review Board of Fukuoka University Hospital (approval number: H19-12-003). Informed consent was obtained in the form of opt-out on the web-site of Fukuoka University Hospital.

Open Access Statement: This is an Open Access article distributed in accordance with the Creative Commons Attribution-NonCommercial-NoDerivs 4.0 International License (CC BY-NC-ND 4.0), which permits the noncommercial replication and distribution of the article with the strict proviso that no changes or edits are made and the original work is properly cited (including links to both the formal publication through the relevant DOI and the license). See: https://creativecommons.org/licenses/by-nc-nd/4.0/.

\section{References}

1. Varela G, Thomas PA. Surgical management of advanced non-small cell lung cancer. J Thorac Dis 2014;6 Suppl 2:S217-23.

2. Borri A, Leo F, Veronesi G, et al. Extended pneumonectomy for non-small cell lung cancer: morbidity, mortality, and long-term results. J Thorac Cardiovasc Surg 2007;134:1266-72.

3. Weder W, Inci I. Carinal resection and sleeve pneumonectomy. J Thorac Dis 2016;8:S882-8.

4. Raveglia F, Baisi A. Carinal sleeve pneumonectomy: oncological recommendations. Shanghai Chest 2017;1:62.

5. Dindo D, Demartines N, Clavien PA. Classification of surgical complications: a new proposal with evaluation in a cohort of 6336 patients and results of a survey. Ann Surg 2004;240:205-13.

6. Abbott OA. Experiences with the surgical resection of the human carina, tracheal wall, and contralateral bronchial wall in cases of right total pneumonectomy. J Thorac Surg 1950;19:906-22.

7. Barclay RS, McSwan N, Welsh TM. Tracheal reconstruction without the use of grafts. Thorax 1957;12:177-80.

8. Grillo HC. Carinal reconstruction. Ann Thorac Surg 1982;34:356-73.

9. Porhanov VA, Poliakov IS, Selvaschuk AP, et al. Indications and results of sleeve carinal resection. Eur J Cardiothorac Surg 2002;22:685-94.

10. de Perrot M, Fadel E, Mercier O, et al. Long-term results after carinal resection for carcinoma: does the benefit warrant the risk? J Thorac Cardiovasc Surg 2006;131:81-9.

11. Rea F, Marulli G, Schiavon M, et al. Tracheal sleeve pneumonectomy for non-small cell lung cancer (NSCLC): short and long-term results in a single institution. Lung Cancer 2008;61:202-8.

12. Eichhorn F, Storz K, Hoffmann H, et al. Sleeve pneumonectomy for central non-small cell lung cancer: indications, complications, and survival. Ann Thorac Surg 2013;96:253-8.

13. Shin S, Park JS, Shim YM, et al. Carinal resection and reconstruction in thoracic malignancies. J Surg Oncol 2014;110:239-44.

14. Dartevelle PG, Mitilian D, Fadel E. Extended surgery for T4 lung cancer: a 30 years' experience. Gen Thorac Cardiovasc Surg 2017;65:321-8.

15. Galetta D, Spaggiari L. Early and long-term results of tracheal sleeve pneumonectomy for lung cancer after induction therapy. Ann Thorac Surg 2018;105:1017-23.

16. Costantino CL, Geller AD, Wright CD, et al. Carinal surgery: A single-institution experience spanning 2 decades. J Thorac Cardiovasc Surg 2019;157:2073-83.e1.

17. Depierre A, Milleron B, Moro-Sibilot D, et al.

Preoperative chemotherapy followed by surgery compared with primary surgery in resectable stage I (except T1N0), II, and III a non-small-cell lung cancer. J Clin Oncol 2002;20:247-53. 
18. Tada H, Tsuchiya R, Ichinose $\mathrm{Y}$, et al. A randomized trial comparing adjuvant chemotherapy versus surgery alone for completely resected pN2 non-small cell lung cancer (JCOG9304). Lung Cancer 2004;43:167-73.

19. Martin J, Ginsberg RJ, Abolhoda A, et al. Morbidity and mortality after neoadjuvant therapy for lung cancer: the risks of right pneumonectomy. Ann Thorac Surg 2001;72:1149-54.

doi: $10.21037 /$ shc.2020.03.06

Cite this article as: Waseda R, Miyahara S, Moroga T, Sato T, Shiraishi T, Iwasaki A. Elucidation of clinicopathological factors associated with improved clinical outcomes for carinal pneumonectomy in T4 non-small cell lung cancer. Shanghai Chest 2020;4:38.
20. Casiraghi M, Maisonneuve P, Piperno G, et al. Salvage surgery after definitive chemoradiotherapy for nonsmall cell lung cancer. Semin Thorac Cardiovasc Surg 2017;29:233-41.

21. Bott MJ, Yang SC, Park BJ, et al. Initial results of pulmonary resection after neoadjuvant nivolumab in patients with resectable non-small cell lung cancer. J Thorac Cardiovasc Surg 2019;158:269-76. 